\title{
Auǵmentation du nombre d'appels relatifs à une exposition à certains nettoyants et désinfectants au début de la pandémie de COVID-19 : données des centres antipoison canadiens
}

\author{
Abdool Yasseen III, Ph. D. (1); Deborah Weiss, Ph. D. (1); Sandy Remer, M.D. (1,2); Nina Dobbin, M. Sc. (1,3); \\ Morgan MacNeill, M. Sc. (1,4); Bojana Bogeljic, M.A. (1); Dennis Leong, B. Sc. (Pharm.) (3); Victoria Wan, M. Sc. (3); \\ Laurie Mosher, I.A. (4); Guillaume Bélair, M. Sc. (5); Margaret Thompson, M.D. (2); Brooke Button, B. Sc. inf. (6); \\ James Hardy, B. Sc. (7); Shahid Perwaiz, Ph. D. (8); Alysyn Smith, I.A. (8); Richard Wootton, B.A. (1)
}

Publié en ligne le 23 septembre 2020

Diffuser cet article sur Twitter

\begin{abstract}
Résumé
On sait peu de choses sur l'utilisation, correcte ou incorrecte, des produits de nettoyage pendant la pandémie de COVID-19. Nous avons compilé des données provenant de centres antipoison canadiens pour janvier à juin 2019 et janvier à juin 2020 et nous rendons compte ici des appels relatifs à certains produits de nettoyage et de l'évolution en pourcentages entre ces deux périodes. Il y a eu 3408 appels (42\%) portant sur des agents de blanchiment, 2015 (25\%) sur des désinfectants pour les mains, 1667 (21\%) sur des désinfectants, 949 (12\%) sur le chlore gazeux et 148 (2\%) sur la chloramine gazeuse. On a observé une augmentation du nombre d'appels en concomitance avec l'apparition de la COVID-19, avec un pic en mars. L'accès rapide aux données des centres antipoison canadiens a permis une communication précoce de messages de sécurité au public.
\end{abstract}

\section{Introduction}

Le premier cas canadien de la nouvelle maladie à coronavirus 2019 (COVID-19), causée par une infection par le SRAS CoV-2, a été enregistré le 15 janvier 2020. Au moment de la rédaction de ce rapport, en août 2020, 117792 cas de COVID-19 confirmés en laboratoire avaient été signalés, dont 8958 décès ${ }^{1,2}$. En février et mars 2020, les messages des responsables canadiens en santé publique ont été de plus en plus axés sur les stratégies visant à réduire la propagation du SRAS CoV-2, comme la distanciation physique, l'étiquette respiratoire, le lavage des mains, l'utilisation de désinfectants pour les mains à base d'alcool et le nettoyage et la désinfection des surfaces fréquemment touchées ${ }^{3}$. Le 11 mars 2020, l'Organisation mondiale de la santé a officiellement déclaré que l'épidémie de COVID-19 était une pandémie mondiale ${ }^{4}$. Dans les jours qui ont suivi, les autorités fédérales, provinciales, territoriales et municipales du Canada ont mis en œuvre des mesures rigoureuses de distanciation physique, en particulier des restrictions de déplacement, la fermeture temporaire d'entreprises et d'écoles et l'annulation des rendez-vous et des interventions médicaux non urgents. Les messages quotidiens des responsables de la santé publique à tous les échelons de gouvernement ont renforcé l'urgence de prendre des mesures afin de limiter la propagation du SRAS-CoV-2.
Points saillants

- Le Système canadien de surveillance des données sur les intoxications (SCSDI), dirigé par Santé Canada, est un réseau en développement composé de centres antipoison, d'autorités sanitaires et d'organismes de réglementation, qui facilite la détection précoce des incidents d'empoisonnement et une alerte rapide au niveau national afin d'éclairer les interventions en matière de réduction des risques.

- En réponse à la pandémie de COVID19, des préoccupations ayant émergé quant au risque de mauvaise utilisation de produits de nettoyage et de désinfectants, le SCSDI a surveillé et évalué ces préoccupations.

- Une augmentation globale du nombre d'appels concernant plusieurs produits de nettoyage et désinfectants a eu lieu en concomitance avec la pandémie, certaines augmentations pouvant atteindre jusqu'à $400 \%$ pour certains produits par rapport à la même période de l’année précédente.

\section{Rattachement des auteurs :}

1. Division de la gestion des urgences chimiques et de la toxicovigilance, Santé Canada, Ottawa (Ontario), Canada

2. Centre antipoison de l'Ontario, Toronto (Ontario), Canada

3. Centre de contrôle des maladies de la Colombie-Britannique, Drug and Poison Information Centre, Vancouver (Colombie-Britannique), Canada

4. Izaak Walton Killam Poison Centre, Halifax (Nouvelle-Écosse), Canada

5. Centre antipoison du Québec, Québec (Québec), Canada

6. Service d'information sur les poisons et les drogues, Calgary (Alberta), Canada

7. Direction de la sécurité des produits de consommation et des produits dangereux, Santé Canada, Ottawa (Ontario), Canada

8. Direction générale des produits de santé et des aliments, Santé Canada, Ottawa (Ontario), Canada

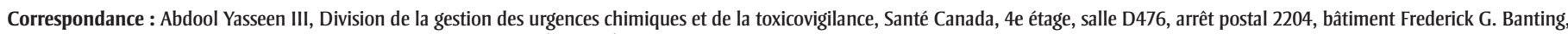
251, promenade Sir Frederick Banting, Tunney's Pasture, Ottawa (Ontario) K1A 0K9; tél. : 343 542-8251; courriel : abdool.yasseen@canada.ca 
Cette augmentation de l'attention portée au nettoyage et à la désinfection est susceptible d'entraîner une exposition accrue aux produits chimiques contenus dans les produits de nettoyage, plus spécifiquement aux fumées et aux sous-produits créés par un mélange inapproprié de ces produits $^{5,6}$. Dans un rapport des US Centers for Disease Control and Prevention, on a signalé une augmentation du nombre d'appels aux centres antipoison relatifs à des expositions à des produits de nettoyage et de désinfection au cours des trois premiers mois de 2020 par rapport à la même période en $2019^{5}$. Bien que l'augmentation de l'exposition ait été signalée au sein de tous les groupes d'âge, l'exposition des jeunes enfants a concerné une grande partie des appels. Les centres antipoison de France ont relevé des questions similaires, émettant l'hypothèse que la présence accrue de jeunes enfants dans les foyers, combinée à des changements de comportement en matière de nettoyage, est susceptible d'entraîner une augmentation des empoisonnements ${ }^{6}$. $\mathrm{Au}$ Canada, on sait peu de choses sur la déclaration de ces produits, surtout en période de pandémie; dans ce rapport, nous présentons les données des cinq centres antipoison du pays.

\section{Méthodologie}

Nous rendons compte des données anonymisées obtenues auprès des cinq centres antipoison canadiens au moyen d'un processus de demande de renseignements, données qui ont été compilées au moyen du programme du Système canadien de surveillance des données sur les intoxications (SCSDI) dirigé par Santé Canada. Ces données rassemblent les renseignements recueillis lors des appels concernant une exposition reçus entre janvier et juin 2019 ainsi qu'entre janvier et juin 2020, en utilisant pour ces expositions la classification de l'American Association of Poison Control Centers (AAPCC), avec comme codes 200613, 200614, 200615 et 200616 pour les désinfectants pour les mains, 39282, 40280, 42281 et 77286 pour les désinfectants, 42280, 62280, 77280 et 77282 pour les agents de blanchiment, 116400 et 116401 pour le chlore gazeux et 77403 pour la chloramine gazeuse. Les appels concernant les expositions sont présentés sous forme d'effectifs globaux et stratifiés par âge de la personne exposée (jeunes de 19 ans et moins et adultes de 20 ans et plus) et ont été évalués mensuellement pour les cinq centres antipoison entre janvier et juin 2019 et 2020, ainsi qu'hebdomadairement pour quatre de ces cinq centres entre mars et avril 2020. Nous avons calculé comme suit la variation en pourcentages des estimations entre les deux années :

$\% \Delta_{\text {entre deux années }}=\frac{\left(\text { Mois }_{2020}-\text { Mois }_{2019}\right)}{\text { Mois }_{2019}} * 100 \%$

Il est à noter que, pour l'un des centres antipoison, les données n'étaient pas disponibles avant le 6 février 2019, ce qui fait que les renseignements présentés au niveau national sont partiellement manquants pour cette période. Nous avons consulté le Comité d'éthique de la recherche (CER) de Santé Canada pour discuter de l'initiative du SCSDI et nous avons conclu qu'un examen par le CER n'était pas nécessaire étant donnée l'exigence de suppression obligatoire des cellules de données avec petits effectifs, ce qui garantit un risque minimal de ré-identification.

\section{Résultats}

Au total, il y a eu 8187 appels signalant des expositions entre janvier et juin 2019 et janvier et juin 2020. Parmi ceux-ci, 3408 appels (42\%) concernaient des agents de blanchiment, 2015 (25\%) des désinfectants pour les mains, 1667 (21\%) des désinfectants, 949 (12 \%) du chlore gazeux et 148 (2\%) de la chloramine gazeuse. Nous avons observé une augmentation de $35 \%$ du nombre total d'expositions liées à ces produits de nettoyage en janvier 2020 par rapport à janvier 2019, mais aucune différence apparente en février. Cependant, en février 2020, nous avons constaté une grande hétérogénéité entre les différents types de produits de nettoyage, les désinfectants pour les mains et les désinfectants ayant chacun augmenté d'environ $40 \%$, et les agents de blanchiment, le chlore gazeux et la chloramine gazeuse ayant diminué de respectivement $3 \%, 9 \%$ et $12 \%$ par rapport à février 2019 (figure 1). Nous avons également observé une augmentation plus marquée pour mars à juin, en particulier en ce qui a trait aux expositions aux désinfectants et à la chloramine gazeuse. D’après les estimations hebdomadaires, le nombre d'appels a atteint un pic pendant la semaine du 22 mars 2020, principalement chez les plus de 19 ans (figure 2) et la variation en pourcentage a diminué de manière générale après avril 2020, à l'exception des désinfectants pour les mains et de la chloramine gazeuse.

\section{Analyse}

En utilisant les données des centres antipoison canadiens, nous avons observé une augmentation des appels concernant l'exposition à certains nettoyants et désinfectants en mars 2020 par rapport à mars 2019. Les données hebdomadaires recueillies pour mars à mai 2020 indiquent que l'augmentation a atteint son maximum au cours de la troisième semaine de mars, peu après que l'OMS ait déclaré que la COVID-19 était une pandémie mondiale. Ces résultats concordent avec les rapports des États-Unis et, compte tenu des circonstances, semblent indiquer une augmentation de l'exposition à ces produits en lien avec le début de la pandémie mondiale de COVID-195. La ou les raisons de cette augmentation ne sont pas claires, mais peuvent être liées à une utilisation accrue de produits de nettoyage visant à essayer d'atténuer les risques d'infection par le coronavirus. En outre, la disponibilité limitée de certains nettoyants pourrait avoir entraîné une utilisation ou un mélange inapproprié d'autres produits, ainsi qu'une mauvaise utilisation de produits pour l'hygiène personnelle ou la décontamination ${ }^{6}$. Avec la fermeture des écoles et des garderies, les jeunes enfants se sont retrouvés à la maison, ce qui peut avoir favorisé les risques d'exposition. Toutefois, nos résultats ne font pas état d'une forte augmentation chez les 19 ans ou moins. Les variations en pourcentage pour janvier et février sont peut-être liées aux préoccupations précédant la pandémie, mais elles sont plus probablement fortuites, car on ne remarque pas de différences majeures.

Nos résultats montrent que les messages proactifs ont vraisemblablement communiqué efficacement les risques éventuels liés aux nettoyants et aux désinfectants pendant la pandémie de COVID-19. En réponse aux données de surveillance précoce, les responsables de la santé publique et les organismes de réglementation ont en effet préconisé une utilisation sûre de ces produits et ont souligné l'importance d'une communication rapide des données des centres antipoison à l'échelle nationale. Après avoir effectué les premières observations en mars 2020, nous avons engagé des discussions avec les cinq centres antipoison et les programmes du gouvernement fédéral canadien chargés d'améliorer la sécurité des produits de nettoyage sélectionnés. Cette rencontre préliminaire a servi de point de départ à la 
FIGURE 1

Nombre d'appels passés à des centres antipoison canadiens concernant certains produits de nettoyage et désinfectants en 2019 et 2020 (janvier à juin), avec variations en pourcentages d'une année à l'autre
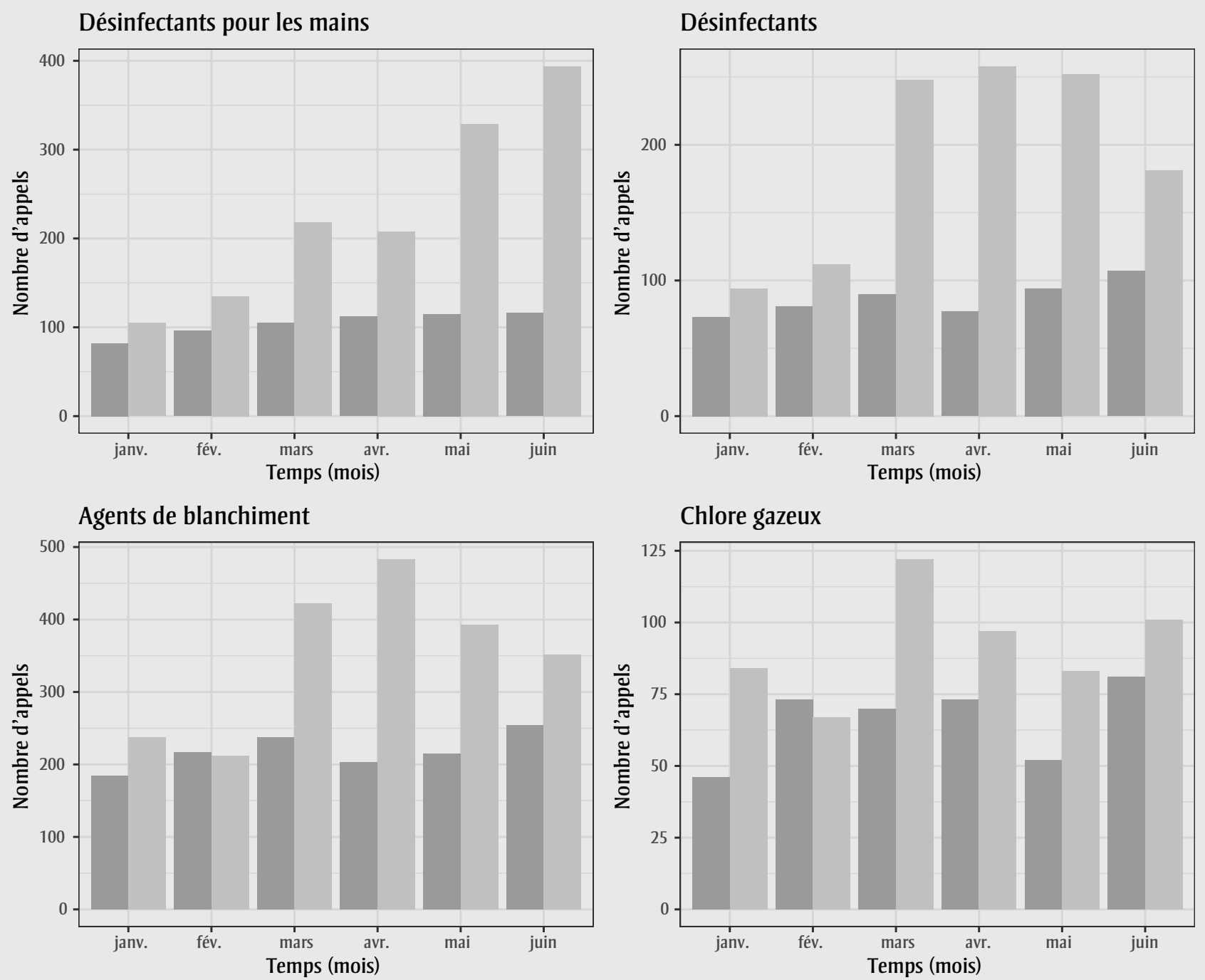

Chloramine gazeuse

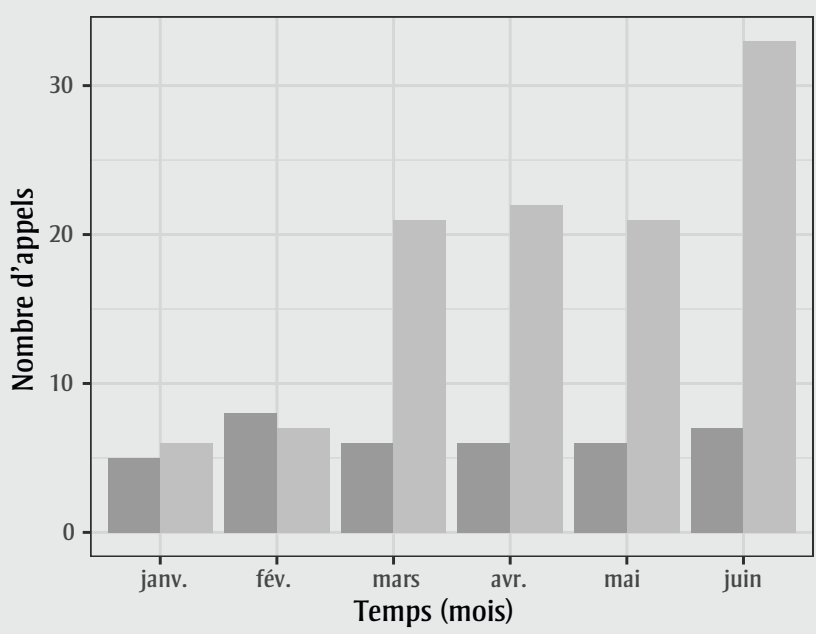

Variation en pourcentage (2020 par rapport à 2019)

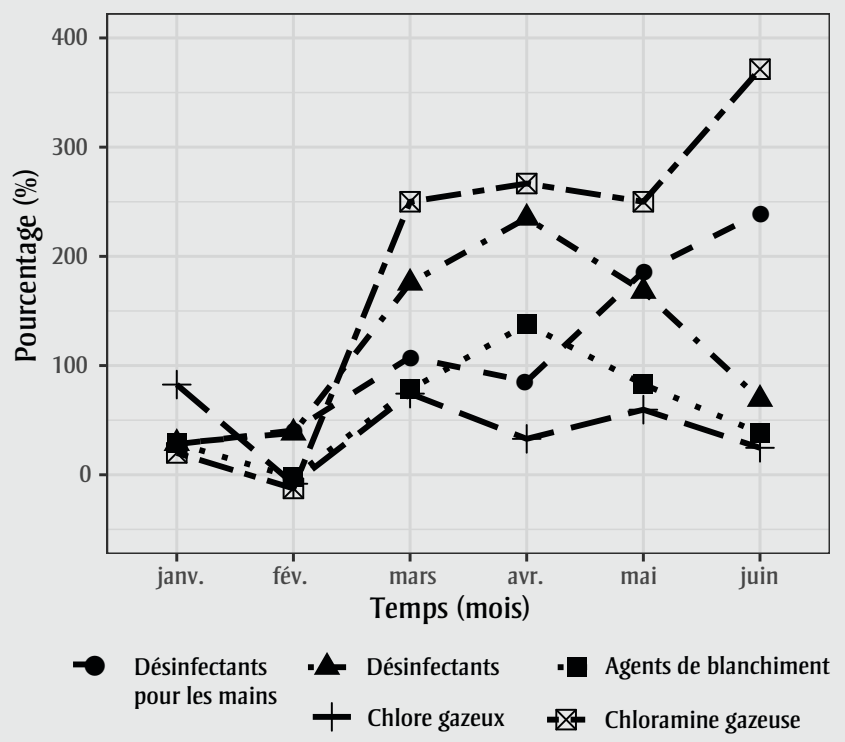


FIGURE 2

Nombre d'appels hebdomadaires concernant une exposition à des produits de nettoyage en 2020, stratifié par groupe d'âge et par produits de nettoyage, pour quatre des cinq centres antipoison canadiens ${ }^{a}$

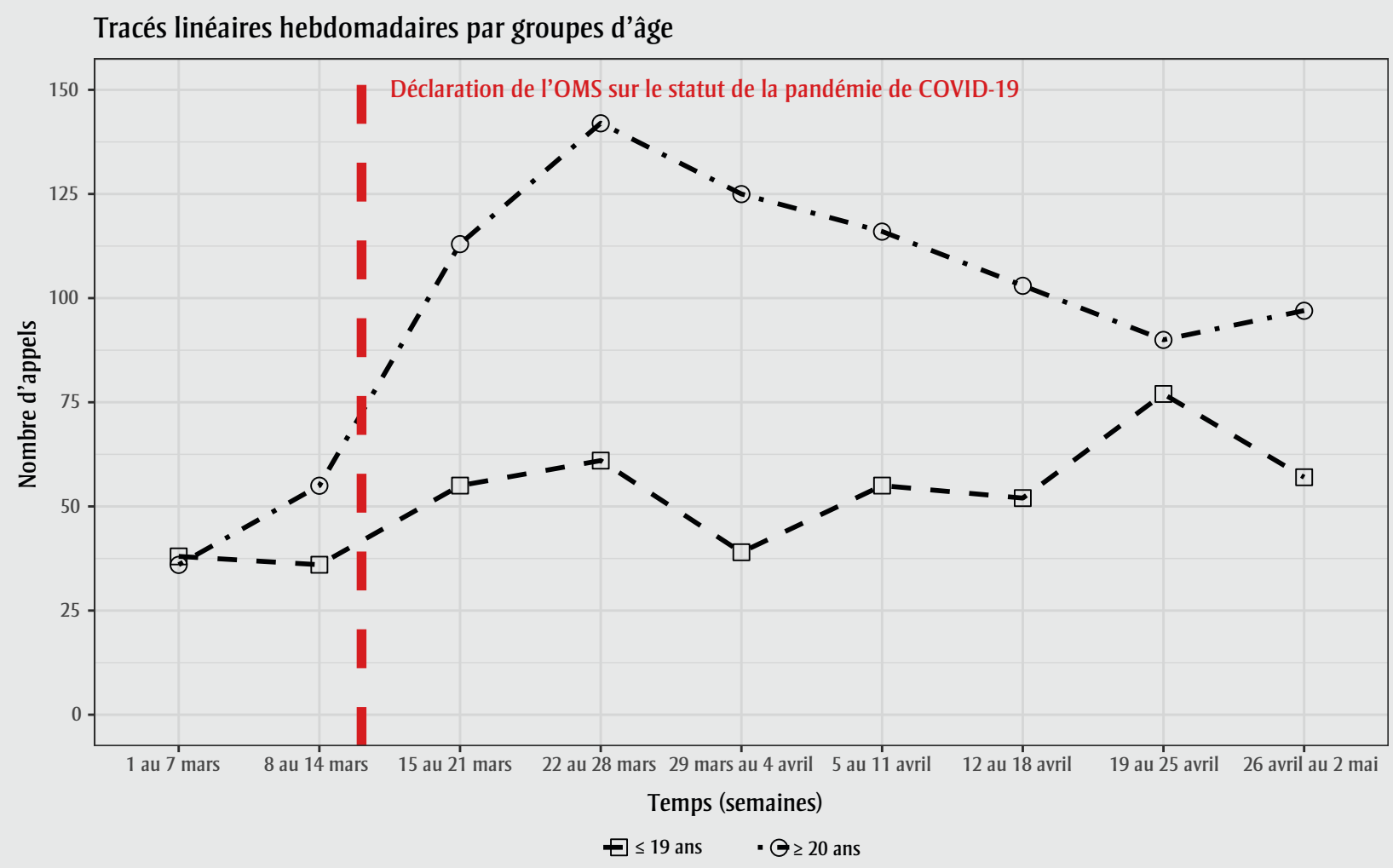

Tracés hebdomadaires par produits de nettoyage et désinfectants

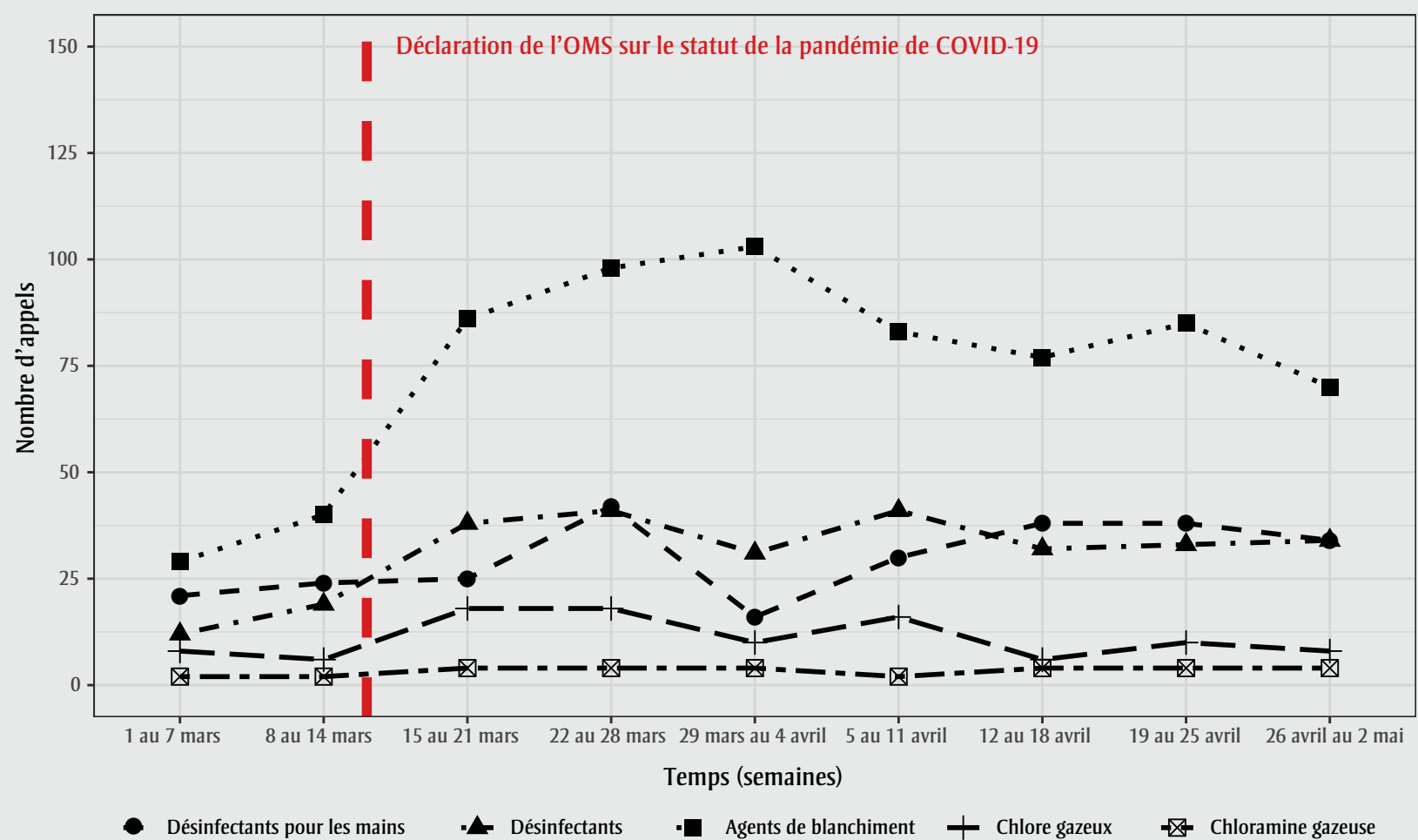

${ }^{a}$ L'un des centres antipoison n'ayant pu fournir de données hebdomadaires, il a été exclu de cette analyse. 
conception des messages (tant pour les médias sociaux que pour la diffusion en ligne) visant à renforcer l'utilisation sécuritaire de ces nettoyants et désinfectants. Entre mars et juin 2020, Santé Canada a déployé un certain nombre de tactiques de communication afin d'informer les Canadiens sur l'utilisation sécuritaire des nettoyants ménagers, des désinfectants et des désinfectants pour les mains, en particulier des campagnes simultanées dans les médias sociaux diffusées par divers canaux ministériels sur Twitter, Facebook et LinkedIn. Le contenu pour ces médias sociaux encourageait le lavage des mains et le nettoyage des surfaces, et fournissait des renseignements sur la manière de le faire en toute sécurité. Les médias sociaux ont également relayé l'information sur les risques liés à la fabrication de désinfectants pour les mains à la maison et au mélange de produits de nettoyage, ainsi que sur l'utilisation sécuritaire de l'eau de Javel et l'importance de tenir ces types de produits hors de portée des enfants. Outre la diffusion auprès des médias sociaux, Santé Canada a créé un portail Internet (https://www.canada.ca/fr/sante-canada /services/medicaments-produits-sante /desinfectants/covid-19.html) consacré aux désinfectants et aux produits d'hygiène pour les mains. Ce site est régulièrement mis à jour et contient des renseignements sur les mesures prises par le gouvernement du Canada pour accroître l'approvisionnement de ces produits, ainsi que sur la manière de les utiliser en toute sécurité. De plus, Santé Canada a publié divers avis publics et communiqués de presse sur l'importance de nettoyer, désinfecter et assainir les mains et les surfaces en toute sécurité.

\section{Points forts et limites}

L'un des principaux points forts de ce projet est qu'il combine les données régionales et l'expertise des centres antipoison canadiens pour fournir des statistiques nationales à l'échelle du Canada. Nous avons diffusé des versions préliminaires de ce rapport par l'intermédiaire du réseau du SCSDI, qui regroupe des spécialistes cliniques, des responsables de la santé publique et du personnel des organismes de réglementation, afin d'élaborer collectivement des lignes directrices et de permettre l'application des connaissances et l'échange de messages sur l'utilisation appropriée de ces produits. En outre, ce rapport utilise certains renseignements des centres antipoison qui ne sont pas disponibles auprès d'autres sources de données administratives sur la santé et qui demeurent une ressource relativement inexploitée pour comprendre l'épidémiologie des expositions environnementales au Canada.

Cependant, comme pour toute donnée secondaire, il existe certains biais potentiels susceptibles d'avoir une incidence sur l'interprétation des résultats observés. Nos analyses sont basées sur les appels à des centres antipoison canadiens et ne représentent probablement qu'une petite partie des expositions totales, car certaines personnes se prendront en charge à la maison tandis que d'autres iront directement chercher des soins médicaux en personne. En outre, les spécialistes de l'information antipoison des centres antipoison se fondent sur les réponses fournies par les appelants pour traiter ou gérer les expositions indésirables, ce qui fait que les renseignements consignés dans leurs systèmes de gestion des cas dépendent de la capacité de l'appelant à décrire précisément l'exposition subie. La plupart des régions du Canada disposent d'un centre antipoison mais pas toutes, ce qui fait que les données ne sont pas pleinement représentatives au niveau national. Cependant, l'information fournie ici constitue la meilleure estimation disponible au moment où nous publions. Par ailleurs, l'historique des données sur les produits de nettoyage et les désinfectants avant 2019 n'était pas disponible au moment de la publication, ce qui fait que nous n'avons pas pu réaliser d'analyse statistique plus poussée. De plus, malgré les efforts en cours visant à améliorer la qualité et la comparabilité des données des centres antipoison, il demeure des incohérences dans la codification à la fois au sein des centres antipoison et entre eux. Enfin, ces résultats montrent certes une corrélation entre l'augmentation du nombre d'appels aux centres antipoison canadiens concernant une exposition à des produits de nettoyage et des désinfectants et la pandémie de COVID-19 au Canada, mais on ne peut en déduire de lien de causalité étant donné que cette relation n'est pas bien comprise et qu'il y a sans doute encore des facteurs déterminants inconnus qui lui sont associés.

\section{Conclusion}

Une augmentation du nombre d'appels aux centres antipoison concernant certains nettoyants et désinfectants s'est produite en même temps que le début de la pandémie de COVID-19 au Canada. Ce travail souligne l'importance d'un accès rapide aux données de ces centres antipoison à l'échelle nationale car ces appels constituent une ressource, relativement inexploitée, pour comprendre l'exposition des Canadiens à des substances chimiques possiblement dangereuses, d'autant plus que ces renseignements ne sont pas facilement accessibles autrement, par exemple au moyen des sources de données administratives sur la santé.

\section{Remerciements}

Ce travail n'aurait pas été possible sans le travail et le soutien des spécialistes de l'information antipoison et des cinq centres antipoison canadiens. Leurs efforts diligents pour fournir des conseils d'experts au public et aux praticiens cliniques ont facilité la collecte et la consignation des renseignements nécessaires à ces activités de surveillance.

\section{Conflits d'intérêts}

Les auteurs déclarent n’avoir aucun conflit d'intérêt réel ou perçu.

\section{Contributions des auteurs et avis}

AY, DW, SR, ND, MM et RW ont conçu et planifié l'étude et ont élaboré le protocole. AY, DW, SR, ND et MM ont élaboré le plan d'analyse et analysé les données. AY et DW ont rédigé l'article initial, distribué ce projet à SR, ND, MM et RW aux fins d'examen préliminaire, puis à tous les autres auteurs cités pour effectuer des contrôles de la qualité, une évaluation des résultats et une révision du texte. Tous les auteurs ont contribué à la révision critique de l'article tout au long du processus de rédaction et de révision.

Le contenu de l'article et les points de vue qui y sont exprimés n'engagent que les auteurs et ne correspondent pas nécessairement à ceux du gouvernement du Canada.

\section{Références}

1. Gouvernement du Canada. Maladie à coronavirus de 2019 (COVID-19) : Mise à jour quotidienne sur l'épidémiologie [Internet]. Ottawa (Ont.) : Gouvernement du Canada; [modification le 24 août 2020; consultation le 12 août 2020]. En ligne à : https://sante -infobase.canada.ca/covid-19/resume -epidemiologique-cas-covid-19.html 
2. Gouvernement du Canada. Maladie à coronavirus (COVID-19) : Mise à jour sur l'éclosion [Internet]. Ottawa (Ont.) : Gouvernement du Canada; [modification le 4 août 2020; consultation le 12 août 2020]. En ligne à : https:// www.canada.ca/fr/sante-publique /services/maladies/2019-nouveau -coronavirus.html

3. Gouvernement du Canada. Maladie à coronavirus (COVID-19) : Prévention et risques [Internet]. Ottawa (Ont.) : Gouvernement du Canada; [modification le 19 août 2020; consultation le 12 août 2020]. En ligne à : https:// www.canada.ca/fr/sante-publique /services/maladies/2019-nouveau -coronavirus/prevention-risques.html

4. Organisation mondiale de la Santé (OMS). Allocution liminaire du Directeur général de l'OMS lors du point presse sur la COVID-19 - 11 mars 2020 [Internet]. Genève $(\mathrm{CH})$ : OMS; 11 mars 2020 [consultation le 12 août 2020]. En ligne à : https://www.who.int/fr /dg/speeches/detail/who-director -general-s-opening-remarks-at-the -media-briefing-on-covid-19---11 -march-2020

5. Chang A, Schnall AH, Law R, et al. Cleaning and disinfectant chemical exposures and temporal associations with COVID-19-National Poison Data System, États-Unis, 1er janvier au 31 mars 2020. MMWR Morb Mortal Wkly Rep. 2020;69(16):496-498.

6. Le Roux G, Sinno-Tellier S, French Poison Control Centre members, Descatha A. COVID-19: home poisoning throughout the containment period. Lancet Public Health. 2020:5(6):e314. Epub 25 avril 2020. Erratum dans : Lancet Public Health. 4 mai 2020: 5(6):e315. 